"This is the peer reviewed version of the following article: Innes, Kelli, Elliott, Doug, Plummer, Virginia, Jackson, Debra (2017) Emergency department waiting room nurses in practice: An observational study, Journal of Clinical Nursing 27(7-8). which has been published in final form at https://doi.org/10.1111/ jocn.14240. This article may be used for non-commercial purposes in accordance with Wiley Terms and Conditions for Self-Archiving." 


\section{Emergency department waiting room nurses in practice: an}

\section{observational study}

Kelli Innes $\mathrm{RN}, \mathrm{MN}^{1,2}$

Doug Elliott RN, $\mathrm{PhD}^{1}$

Virginia Plummer RN, $\mathrm{PhD}^{2,4}$

Debra Jackson FACN, $\mathrm{PhD}^{3,5,1,6}$

1. Faculty of Health, University of Technology Sydney

2. Faculty of Medicine, Nursing and Health Sciences, Monash University

3. Oxford Institute of Nursing, Midwifery \& Allied Health Research (OxINMAHR), Faculty of Health and Life Sciences, Oxford Brookes University, The Colonnade, Gipsy Lane Campus, Headington, Oxford OX3 OBP

4. Peninsula Health, Hastings Road Frankston, Australia 3199.

5. Health Education England - Thames Valley, 4630 Kingsgate, Cascade Way, Oxford OX4 2SU

6. Oxford University Hospitals NHS Foundation Trust; Oxford Health NHS Foundation Trust. 


\section{ABSTRACT}

\section{Aim}

To identify the activities and behaviours of waiting room nurses in emergency department settings.

\section{Background}

Emergency care has expanded into waiting rooms in some emergency departments. Often viewed as an adjunct to triage, the aim of waiting room nurses is to commence care early, reassess patients and improve communication between patients, families and staff. There is however a paucity of literature relating to waiting room nurses, especially in relation to their current activities and behaviours.

\section{Design and methods}

Part of a larger exploratory sequential mixed methods designed study. This phase used a non-participant observer role to observe waiting room nurses in their natural setting undertaking normal care and responsibilities. One observer, using a tool and reflective journal collected data on participant interactions, processes and practices on eight waiting room nurses over 13 episodes of observation (total 65h:50m) in two emergency departments. Data analysis used descriptive statistics and thematic analysis.

\section{Results}

Participants were observed to anticipate and prioritise to deliver holistic, patient centred care in emergency department waiting rooms. Waiting room nurses had a varied and unpredictable workload, including facilitating the flow of patients from the waiting room. They contributed to patient safety in the waiting room, primarily by reassessing and detecting clinical deterioration.

\section{Conclusion}

Further research into this role is required, including linking efficacy with experience of nurses, impact the role has on patient safety, and patient and family perceptions of the role. 


\section{RELEVANCE TO CLINICAL PRACTICE}

Therapeutic engagement allowed waiting room nurses to reassure and calm patients and families, and deliver holistic, patient centred care. Waiting room nurses contributed to patient safety in the waiting room, by promptly commencing episodes of care in the waiting room and through close monitoring and assessment to detect patient deterioration.

\section{KEYWORDS}

mixed methods, observation, qualitative research, emergency departments, emergency nursing, waiting room 


\section{INTRODUCTION}

In response to increased waiting times, poor patient outcomes (Bernstein et al., 2009) and patient dissatisfaction (Garling, 2008), some emergency departments (EDs) have expanded care to patients while still in ED waiting rooms, prior to being seen by a doctor. One model of this post-triage waiting room-based care is a waiting room nurse (WRN); the role is separate and distinct from the triage nurse, but acts as an adjunct and in concert with the triage role. The scope of practice includes post-triage assessments, commence early interventions, re-assessments for waiting patients and to improve communication for patients and families in the waiting room who have yet to be allocated a treatment space in the ED (Innes, Jackson, Plummer, \& Elliott, 2015).

\section{BACKGROUND}

Current literature on the WRN is limited, and has focused on the technical and operational aspects of the waiting room role, including reporting of tasks and activities performed, and their contribution to decreasing waiting times (Cheng et al., 2013; Considine et al., 2012; Fry \& Jones, 2005; Huang et al., 2013). Despite this emphasis, no clinically significant reductions in overall waiting times, ED length of stay, or number of patients leaving without being seen by a medical officer were found (Cheng et al., 2013; Considine et al., 2012; Fry et al., 2012; Huang et al., 2013).

There is also a paucity of literature relating to other aspects of WRN role, including the reasons for implementation (Innes, Jackson, Plummer, \& Elliott, 2017), and the nurses' skills, knowledge and experience required to effectively and efficiently perform the role. With no exploration of the activities undertaken or the behaviours demonstrated by nurses in this challenging environment to care for patients and families, we sought to address this limitation, by identifying the activities and behaviours of WRNs in ED settings.

\section{METHODS}

\section{Design}

This non-participant observational study is drawn from a larger exploratory sequential mixed methods doctoral study examining the nursing role in ED waiting rooms. Mixed methods research combines two or more different approaches to data collection and analysis in a single study 
(Tashakkori \& Teddlie, 1998), and utilises the strengths while counteracting the weaknesses of the different approaches (Creswell, 2009). Exploratory sequential design is used when little is known about a topic, and places an emphasis on qualitative data followed by the collection of quantitative data to explain and quantify the results (Creswell, 2009).

Using mixed methods, data collected in earlier phases informs each progressive phase. The sequential design used in this study enabled data collected from an integrative literature review and Phase One to inform data collection in this current observational phase (Creswell, 2009; Creswell \& Plano Clark, 2011). The results from the integrative review (Innes et al., 2015) and Phase One (Innes et al., 2017) have been reported elsewhere. The aim of this phase of the research was to identify the activities and behaviours of WRNs in ED settings.

\section{Study setting}

The setting for the study was two Australian EDs. Details of both EDs are presented in Table 1. Both settings had a private, dedicated space for use by participants, close to triage and the waiting room. This space consisted of a desk with a computer and a patient assessment space, one ED had a trolley, while the other had a reclining chair. Both spaces also contained oxygen and suction supplies, emergency airway equipment, blood glucose machines and trolleys with equipment for venepuncture and cannulation. An electrocardiography (ECG) machine and weight scales were located in close proximity in both settings. Standing orders were in place in both settings, in the form of nurse initiated policies. Authorised by medical officers, standing orders provide a guide for appropriate assessment and interventions for a variety of patient presentations (Considine, 2011). In both settings nurse-initiated analgesia administration and x-ray ordering were in use, one ED also had nurse-initiated pathology ordering. Nurses were not directly supervised and determined their own priorities for work. 
Table 1 Description of ED settings

\begin{tabular}{|c|c|c|}
\hline Study setting characteristics & Setting 1 & Setting 2 \\
\hline \multicolumn{3}{|l|}{ Hospital } \\
\hline Type & Secondary referral & Tertiary referral \\
\hline Location & $\begin{array}{l}\text { Metropolitan hospital, } \\
\text { major Australian city }\end{array}$ & $\begin{array}{l}\text { Regional hospital, } \\
\text { Victoria (Australia) }\end{array}$ \\
\hline ED Presentations (2015-16) ${ }^{1}$ & 69289 & 53307 \\
\hline ED Structure ( $n=b e d$ spaces) & 59 & 28 \\
\hline Resuscitation & 4 & 3 \\
\hline Cardiac monitored & 20 & 10 \\
\hline Non-monitored & 0 & 8 \\
\hline Fast Track & 9 & 5 \\
\hline Short stay & 18 & 8 \\
\hline \multicolumn{3}{|l|}{ Nurse Staffing (per shift) } \\
\hline Morning (0700-1530hr) & 18 & 18 \\
\hline Afternoon (1300-2130hr) & 19 & 20 \\
\hline Night (2100-0730hr) & 14 & 16 \\
\hline Varied shift hours ${ }^{2}$ & 3 & 1 \\
\hline
\end{tabular}

Note: ${ }^{1}$ Australian Institute of Health and Welfare $(2016) ;{ }^{2}$ Includes WRN role

\section{Sample and recruitment}

The sample were Registered Nurses working in the WRN role. Purposive sampling was used to recruit participants. Nurse Unit Managers (NUM) from both settings identified Registered Nurses who worked as WRN, and sent an email informing them of the project. The observer gained permission from the NUM to enter the setting to perform the observation on pre-determined dates and times. At the commencement of each observation session was the first time the observer had 
contact with participants. At this time, the observer spoke with the nurse who had been allocated to the WRN role to identify if they were willing to participate in the research and gained consent.

\section{Data collection}

Participant observation was identified as the most appropriate method as it allowed for the WRN to be observed first hand, undertaking their normal responsibilities in their normal setting. This allowed the researcher to see how participants worked within, and related, to the environment in real time (Mulhall, 2003). Referred to as the 'gold standard' in qualitative research (Murphy \& Dingwall, 2007), observation is a systematic approach to data collection, whereby the researcher gathers information by seeing what people actually do, rather than what they report they do (Gold, 1958; Mulhall, 2003). In observation, data is collected through observing practices, responses, behaviours, and through listening and enquiring (Borbasi, Jackson, \& Wilkes, 2005; Jackson, McDonald, Luck, Waine, \& Wilkes, 2016). Observation is, therefore, an ideal method for exploring the activities and behaviours of WRN in the clinical setting, as in-depth insights and understandings could be gained (Morse, 2003).

Data collection was performed by the first named author. Gold (1958) identifies two approaches to the non-participant observer role. In this study 'observer as participant' was the nonparticipant observer method adopted by the researcher, as, unlike the 'complete observer', it enables social interaction with participants (Gold, 1958). As a non-participant observer, the observer was an independent and non-judgemental outsider to the group. Although not part of the group and only having brief interactions with participants, the observer was able to interact and converse with participants, and could step in and out of the group as needed (Schneider, Whitehead, LobiondoWood, \& Haber, 2014). This was due to the observer being an experienced, triage prepared ED nurse, familiar with, and having knowledge and experience of the ED setting and activities (Turnock \& Gibson, 2001), enabling the observer to be accepted in to the setting (Bonner \& Tolhurst, 2002) and draw on their expertise and experience to interpret the activities and behaviours of participants. Observation was overt, with participants being aware of the aim of the study and that they were being observed (Turnock \& Gibson, 2001). 
Observational data recorded participant interactions, processes and practices with patients, family and staff. In-the-moment clarifying conversations with participants were also utilised. Conversations allowed the observer to validate their observations to ensure situations were interpreted fairly and appropriately, and to clarify and elaborate on responses from participants (Schneider et al., 2014). The conversations occurred in areas of the ED free of patients, predominantly in the WRN space or the triage area, after the participant had attended to all patient care needs, and prior to commencing an interaction with another patient. Conversations occurred over periods of five to 10 minutes at a time on multiple occasions during each observation session. Conversations were recorded in field notes and were not audio recorded.

Observation and conversations occurred over the period WRN were in operation; limited in both EDs to $1200-2000 \mathrm{hr}$ and $1000-1900 \mathrm{hr}$, to coincide with local peak patient presentations. No observations therefore occurred between $2000 \mathrm{hr}-1000 \mathrm{hr}$. In total, observations and conversations occurred with eight nurses over thirteen episodes between June and November 2016 (Table 2). Data saturation was reached during these observation periods, with no new activities or interactions observed and repetition of field note entries (Boddy, 2016).

\section{Table 2 Description of observations}

Observations

A June-July 2016

8

6

$12.00-20.00$ hours

$4: 00 h-5 h: 25 m$

4h:40m

$37 h: 25 m$
Site

B

November 2016

5

2

$10.00-19.00$ hours

$3 h: 30 m-6 h: 25 m$

5h:41m

28h:25m 
A combination of structured and unstructured observations was used to examine participant activities and behaviours. Structured observation used a systematic approach to collect data on specific aspects of the role, including medication administration, interventions and diagnostics. Unstructured observation allowed the observer to use free text to record notes on observations of participant interactions with staff and those in the waiting room, quotes from participants during the conversations and the observer's reflections whilst in the setting in real time (Turnock \& Gibson, 2001). Further reflections on observations were also entered into a field diary at the end of each session (Mulhall, 2003).

To allow for the recording of structured and unstructured data an observation tool, informed by critical appraisal of the literature and results from key informant interviews, was developed. Two expert emergency nurses, who had experience with WRN operating in their ED, reviewed the observation tool to determine face validity (Schneider et al., 2014). A pilot study, comprising of a four hour observation period in the clinical setting, was used to test feasibility, to identify any ambiguities and misunderstandings associated with the observation tool (Schneider et al., 2014). Based on feedback the observation tool was revised to improve flow and terminology was changed to ensure clarity.

The observation tool contained four sections for recording data: 1) episodes of communication with patients, triage nurses, nurse-in-charge, allied health, medial officers, attendants and family/carers; 2) documentation performed by WRN including with patients, medication charts, triage nurse, nurse-in-charge, allied health, medical officer and information technology systems, as well as episodes of WRN performing triage; 3) interventions and diagnostics completed by WRN [eight medications with a space for 'Medication - other' and 18 interventions/diagnostics were listed]; and 4) free notes and reflections throughout the observation. Space was also available in each of the first three sections for free notes (Supplementary File 1). A separate observation tool was completed for each observation period.

\section{Data Analysis}

Data collected from the observation tool and diary were analysed using descriptive statistics and thematic analysis. Descriptive statistics, reported as frequencies and percentages, were initially 
used to summarise observable activities such as medications administered and interventions and diagnostics performed by participants. All qualitative data from observation, reflection and conversations, including direct quotes, were viewed as one data set for analysis. Hand written notes were typed and then analysed using a six phase thematic analysis framework outlined by Braun and Clarke (2006). Initially, familiarity was established by reading and re-reading the data set. Data were then coded by identifying similar phrases or words. Themes were then formed by collating codes and re-checked across the entire data set for relevance to the research aim. Finally, themes were refined and named before being written up. Themes were identified through an iterative process, with initial analysis conducted by the first named author. Co-authors then independently reviewed the data set and collectively agreed on the identified codes and themes (Braun \& Clarke, 2006).

\section{Ethical Considerations}

The study was approved by the HREC of both health services and the supporting university as low risk, in accordance with the requirements of the National Standard on Ethical Conduct in Human Research 2007 (National Health and Medical Research Council, 2007). In line with HREC approval an email was sent from NUM to participants with a participant information form attached outlining the purpose, potential risks and benefits of the research, and that participation was voluntary with no repercussions if they chose not to participate. Contact details of the research team and Human Research Ethics Committee (HREC) complaints officer were also included. The NUM had no further involvement in the research and were not aware of which nurses participated or did not participate, therefore there was no potential for coercion or perceptions of constraint by potential participants.

Given the low level of risk for participants (National Health and Medical Research Council, 2007, Guideline 2.2.5a), and in line with HREC approval, informed verbal consent was obtained from each participant at the beginning of each observation session. In a private location a conversation took place between the observer and the participant. Participants were reminded of the aim of the research, offered a printed copy of the participant information form to keep, advised that participation was voluntary and the session could end at any time they requested. Participants were informed that the observer would be taking notes using an observation tool to record interactions, processes and 
practices with patients, family members and staff, and from clarifying conversations. One WRN declined to participate and no complaints were received. Patients were considered part of the environment for this project, therefore consent from patients was not required.

\section{RESULTS}

Through conversation it was ascertained that emergency nursing experience of the participants varied from three years to greater than 15 years, with six participants having completed or currently undertaking postgraduate emergency nursing qualifications. All participants were triage prepared. Two participants worked only as a WRN, while the remaining six participants rotated through the position. Two participants had limited experience in the role, with one participant observed in their first shift as the WRN and another having less than two months' experience. All participants were female.

Results of descriptive statistics showed that various forms of analgesia, paracetamol $(n=31)$, non-steroidal anti-inflammatory medications $(n=18)$ and oral opioids $(n=10)$, were the most common medications administered (Table 3).

\section{Table 3 Oral medications administered}

\begin{tabular}{lc}
\hline \hline Medication & Frequency \\
\hline Paracetamol & 31 \\
Non-steroidal anti-inflammatory & 18 \\
Opioids (Endone) & 10 \\
Ondansetron & 4 \\
Panadeine or Panadeine Forte & 4 \\
Bronchodilator & 2 \\
Antihistamine & 1 \\
Aspirin & 1 \\
Diazepam & 1 \\
Prednisolone & 1
\end{tabular}


The most common interventions and diagnostics performed were intravenous (IV) cannulation $(n=34)$, recording an ECG $(n=26)$ and urinalysis $(n=14)$ (Table 4).

Table $4 \quad$ Interventions and diagnostics performed

\begin{tabular}{lc}
\hline \hline Intervention & Frequency \\
\hline Intravenous cannulation (including pathology) & 34 \\
Electrocardiogram & 26 \\
Urinalysis & 14 \\
Venepuncture (including pathology) & 7 \\
Wound care & 4 \\
Commence oral rehydration therapy & 3 \\
Rest, Ice, Compression and Elevation (RICE) of injury & 3 \\
Blood cultures & 1 \\
Application of a sling & 1 \\
Venous blood gas sample & 1 \\
Wound swab & 1 \\
\hline
\end{tabular}

Results of the thematic analysis are presented under four identified themes: Experience of nurse positively affected patient care; Unpredictable workload coupled with diverse patient care needs; Delivering patient centred care; and Identified delays and resource limitations. These themes are discussed in detail below.

\section{Experience of nurse positively affected patient care}

Although all participants were considered to be experienced emergency nurses, they were not all experienced in the WRN role. The two least experienced WRN, as outlined above, were observed to be less confident in prioritising care and were unsure of underpinning policy and processes, seeking clarification from other staff within the ED. This potentially contributed to them 
being less efficient in the role. During conversation two participants discussed their view that efficiency of the role was impacted by the experience of the WRN.

During a further conversation, one participant expressed their perception that triage prepared nurses were more effective in the role, stating they had "better decision making skills and don't need to be delegated tasks by the triage nurse" (P4). Additionally, during the conversation, the participant also expressed that they felt triage prepared nurses in the WRN role were a resource for less experienced triage nurses, such as confirming a triage category and appropriate management of patients. Assisting as a resource to triage was observed on two occasions. An observed example was when one participant, after being asked to administer analgesia to a patient, identified that a trauma patient had been allocated a triage category that did not reflect their urgency of care. The participant worked with the triage nurse to identify clinical descriptors and an appropriate triage category as per the Australasian Triage Scale (Australasian College for Emergency Medicine, 2013), as well as analgesia options for optimal patient outcomes. This intervention resulted in the triage nurse escalating patient care and communicating with the nurse-in-charge to arrange an appropriate cubicle for the patient to be transferred due to their risk of clinical deterioration. In one ED however this ability to triage was a challenge, with one participant noting that WRN were "not meant to triage" (P2). On occasions this conflicted with patient needs, especially when patients experienced delays being triaged. Despite triage not being a formal, sanctioned part of the role in one setting, it was observed that participants in both settings assisted with triage when available to do so.

Clear evidence of clinical decision-making was observed; the nurses with experience in the WRN role $(n=6)$ independently anticipated and prioritised care needs and interventions for patients in the waiting room, including paediatric patients and families. Importantly, these participants were observed to implement a holistic approach to patient care, anticipating patient care needs including care that fell outside nurse-initiated policies. Examples of this included adding requests for venous blood gases and blood cultures when other pathology was being taken and making referrals early to allied health services.

\section{Unpredictable workload coupled with diverse patient care needs}


Workload for participants was observed to be variable and unpredictable; at times there were no patient care needs in the waiting room, while on other occasions care demands exceeded the capacity for a single WRN. On one occasion when the waiting room became overcrowded, a second nurse was sent to assist the allocated WRN to manage the workload.

Care needs for patients in the waiting room were diverse and variable. Focused patient assessments relating to their presenting condition were frequently observed. It was noted that participants had longer time to assess and gather information on the presenting condition and history compared to triage nurses. On one observed occasion, this additional information resulted in a change to a patient's clinical urgency and re-allocation of triage category.

Reassessing and monitoring patients was an important aspect of the role and contributed to ensuring patient safety in the waiting room. This was highlighted when participants were observed to detect six episodes of patient deterioration. These patients were immediately re-assessed and prioritised with appropriate escalation of care implemented. Deterioration was detected in patients brought into WRN space as well as those in the waiting room. On one occasion, a participant identified and prioritised care of a patient 'slumped' in a chair in the waiting room.

In both settings, participants reported they could only administer IV fluid or nitrates to patients in the waiting room if they had permission from the nurse-in-charge as a "last resort with the preference to find a cubicle in the department" (P1), due to safety concerns, need for close patient monitoring and risk of patient deterioration. It was observed on one occasion that IV fluids were administered to a patient allocated to the waiting room. After initially assessing the patient, the WRN requested a medical review where the decision was made to commence IV fluids. As there were no cubicles available in the ED, the participant commenced the fluids in the corridor within close proximity to WRN space. After this episode of care, a conversation with the participant revealed the decision to care for the patient in the corridor was made on an assessment that there were low patient safety risks and concerns, and this would keep the WRN space free to continue seeing patients.

It was observed that nurses in both settings, assisted with the allocation and flow of patients from the waiting room into a cubicle, effectively acting as an 'intake' nurse. Participants in conversation stated this occurred to circumvent normal channels of calling for attendants, who may 
not be available, or directing patients to find cubicles, which can be challenging due to the layout of the ED or language barriers. On several occasions, participants actually commenced assessment of patients once in the cubicle; in particular, patients with time-critical conditions such as chest pain, if another nurse was not immediately available. After seeking further clarity, participants stated they saw this as an appropriate aspect of their workload as it meant that essential care was not delayed.

\section{Delivering patient centred care}

A holistic patient centred care approach to patient care was observed. This was evidenced through all participants, experienced triage prepared emergency nurses, meeting the physical, emotional, social and spiritual needs of patients and their families. Patients' physical needs were met through assessing and commencing interventions to manage their presenting condition such as administering analgesia for pain or antiemetic for nausea.

Central to the emotional care of patients and families was the development of therapeutic relationships and communication. Participants were observed to make eye contact, introduce themselves and explain their role to patients and families. Patient centred care was delivered by participants being respectful, empathetic and sincere when listening to patient histories. Participants ensured that patients and families were involved with all discussions and decisions relating to their presentation and plan. Participants were observed to clarify unclear points and used language and terminology appropriate for specific patients. This ensured that rapport was established quickly with patients and families, across all age groups.

Participants also explained the WRN role and informed patients and families of ED processes, which contributed to addressing issues such as uncertainty and distress. During one conversation, a participant explained to a patient and their family that she "was going to get things started and hopefully results would be back by the time they saw a medical officer" (P7). The participant stated that she "gets no backlash from patients" (P7), as she clearly communicates the role and advises "people of delays" (P7). Alleviating uncertainty and distress was also observed through participants requesting that patient and families let them know if they became more unwell or had concerns while waiting. 
Another observed example of meeting the emotional needs of patients was when a participant identified a patient experiencing mental health difficulties who had become restless and agitated in the waiting room. The participant engaged with the patient to assess and assist them with managing their distress and anxiety, effectively demonstrating de-escalation as a therapeutic communication technique.

Spiritual needs of patients and families were observed to be met through the delivery of culturally competent care. The WRN was observed to make referrals and collaborate with healthcare workers in their organisation who could ensure that social, cultural and linguistic needs of patients were met. An example of this was participants referring patients to the Indigenous liaison worker.

Disseminating information and discussing management of illness or injuries at home was observed. For example, a patient had returned to the ED to have his burns reviewed as he was unsure about their ongoing management. The participant reassessed the burns and reassured the patient that there were no complications and then proceeded to discuss the application of the cream, specifically addressing patient questions and concerns.

A high level of collaboration with triage nurses was also evident and helped ensure appropriate and timely care was delivered to patients in the waiting room. Frequent handover and updates were observed, with a focus on ascertaining if there were any urgent care needs for recently triaged patients. During a busy period, one participant was observed to start a written list of patient care needs that the triage nurse added to. Other forms of written communication observed were documenting in patient ED charts, medication charts and electronic medical records. Participants were also observed to collaborate with midwives, medical staff, allied health, pharmacy, security, ambulance, mental health liaison, pathology and clerks to deliver patient centred care in ED waiting rooms.

An observed challenge to communication was the noisy environment. In one ED it was observed that the triage, waiting room and WRN space was a relatively open space, with clerks, doctors and security present. Multiple conversations therefore made it difficult for participants, patients and families, to concentrate and hear when interacting.

\section{Identified delays and resource limitations}


Participants were observed to experience a number of delays and time away from the waiting room area. One observed factor contributing to delays was medical officers often being difficult to locate and not immediately accessible to review ECGs or discuss management plans for example. Not having nurse-initiated pathology ordering caused additional delays and frustration for participants in one ED. Despite having identified which bloods tests were required, participants had to locate a medical officer to order the pathology. On an ad hoc basis, a rapid assessment medical officer was allocated in one of the EDs, which improved access and efficiency.

Conversely medical officers occasionally experienced delays accessing the WRN, also leading to delays and potentially compromising safety for patients in the waiting room. It was observed that intermittently during busy periods, both participants and medical officers were assessing and commencing management for patients in separate spaces. If the WRN was unavailable the medical officer would return patients to the waiting room with no handover, resulting in participants "needing to be a detective" (P4) to locate paperwork and determine management plans.

Environment and other staff practices also contributed to delays. Although some patient assessment and interventions could be undertaken in the waiting room, privacy issues and access to appropriate equipment resulted in the majority of patients being taken into the WRN space. Although, as previously presented, the WRN space was often used by medical officers, without consultation with the WRN, to assess and manage patients when the department was busy and overcrowded. Use of this space, often for extended periods of time prevented the nurses from continuing to assess, commence interventions and review some patients.

\section{DISCUSSION}

Key findings from the study were that experienced, triage prepared emergency nurses had the requisite knowledge and skills to effectively perform the WRN role. Therapeutic engagement allowed WRN to deliver a holistic, patient-centred approach to care, support and inform patients and families in ED waiting rooms. WRN facilitated the flow of patients from the waiting room into the ED, and through close engagement and assessment provided an important clinical safety net for these patients. 
There was a sense from three participants that professional experience potentially influenced the ability of nurses to optimally perform the role. The ED waiting room is often a challenging environment to provide safe, effective care to patients and families (Garling, 2008). It is therefore not unexpected that the perception was that experienced emergency nurses were better equipped to perform the role. With all participants being considered experienced emergency nurses, they were more likely able to deal with the rapidly changing, busy, and often stressful environment (Smyth \& McCabe, 2016). Experienced nurses are likely to have greater in depth knowledge, possess critical thinking skills, and are able to assimilate all aspects of assessment, evidence based practice and past experience in clinical making decisions (Odell, 2015). In our study, participants with more experience in the WRN role were observed to be flexible, proactive and demonstrated their initiative by identifying and anticipating patient care needs that fell outside nurse-initiated policies. As such, these participants demonstrated an ability to take control, seize opportunities and anticipate problems (Rehnström \& Dahlborg-Lyckhage, 2016); clearly valuable attributes when providing care in the unpredictable environment of the ED waiting room.

It was evident in our study that participants brought a patient-centred and holistic approach to their caring practice for patients and families in ED waiting rooms. The ability to develop therapeutic nurse-patient relationships allowed WRN to deliver responsive and compassionate nursing care. An aspect of developing therapeutic relationships was that participants were engaged, present and available, and demonstrated care in their actions and interactions (Luck, Jackson, \& Usher, 2009) and were empathic to the perceived needs and concerns of patients and families (Cecil \& Glass, 2015). This approach allowed participants to offer comfort and information, to calm and reassure patients and families (Luck et al., 2009). Respect and trust was established by participants through their interactions with patients and families, which were adapted to best meet the needs of individual patients and families.

Participants used appropriate language and non-verbal communication, and remained calm and positive, therefore providing reassurance to patients and families and contributed to the creation of safe and secure therapeutic environment (Luck et al., 2009). Their skill in doing this was valuable, as establishing therapeutic relationships can be challenging in this context, where patients and families are often stressed, distressed and anxious due to illness, long waiting times and lack of 
communication whilst waiting, as well as the hectic, noisy environment in which they find themselves (Kamali, Jain, Jain, \& Schneider, 2013; Luck et al., 2009; Welch, 2010). Findings of this study are supported by Fry et al. (2013) who found that delivery of compassionate care was central to the effective implementation of the Clinical Initiative Nurse practice, one model of a WRN role.

WRN can play an important role in patient safety by contributing to the delivery of safe, quality healthcare. It is widely documented that long waiting times and delays in receiving treatment in EDs negatively impacts patient safety and outcomes (Burke et al., 2017). EDs that allocate a WRN shift a patient's episode of care from commencing once in a cubicle, which may not occur for many hours, to effectively commencing on their arrival to the ED (aside from the triage process). Therefore, potentially improving the quality and safety of the waiting experience for patients and families in the waiting room, compared to EDs that do not have an equivalent role.

Involving patients and families in discussions and decisions on their health also contributed to WRN influencing patient safety (Australian Commission on Safety and Quality in Health Care, 2012) and by co-operating and interacting with the interprofessional healthcare workforce (Australian Commission on Safety and Quality in Health Care, 2012). Successful interactions in this context require mutual understanding, respect and trust between team members (Clark, 2009). This can be challenging in the ED due to the unpredictable nature of the work and patient presentations, the time constrained environment and frequently changing team members (Friberg, Husebø, Olsen, \& Sætre Hansen, 2016).

On-going assessment and monitoring of health status of waiting patients also contributed to patient safety. One study found that deterioration and response to interventions could be detected if patients were monitored in ED waiting rooms. It must be noted that the study used a wireless vital sign monitoring device on patients in the waiting room (Hubner et al., 2015). In this study, two factors were identified that affected WRN ability to monitor patients. Firstly, the re-allocation of WRN to assist with other patient care needs in the ED. We assert that during busy periods, when waiting times are extended, WRN is most needed in the waiting room due to greater numbers of patients, longer waits and increased risk of unnoticed patient deterioration (Garling, 2008). Secondly, the limited hours of operation of WRN, means there is no allocation overnight. Patient safety in the waiting room during the hours that the WRN is not in operation needs to be considered, especially 
during periods when there is decreased flow due to access block and boarding issues as a result of limited access to hospital beds (Mason, Knowles, \& Boyle, 2017). This, coupled with decreased resources on night duty (Australasian College for Emergency Medicine, 2016), may result in an increased risk to patient safety. Not with standing this, decreased patient presentations overnight may mitigate some risk to patient safety. Further evidence evaluating the impact of WRN on patient safety is needed.

Patient safety was also influenced by participants who were observed to contribute to enhanced health literacy, through informing patients about strategies for managing their health, once discharged. Health literacy is how people understand and apply information and use it to make decisions about their health and health care. Improving health literacy contributes to decreasing adverse outcomes, therefore improving outcomes for patients and communities (World Health Organization, 2013).

Assisting with flow of patients out of the waiting room into ED cubicles was a previously unreported aspect of workload for our participants. The observed practice was aimed at limiting or decreasing length of stay during patients' transitions through the ED (Asplin et al., 2003). On face value, the use of WRN to transfer patients from the waiting room, could be considered as being contributory to improving the overall efficiency of the ED; in that time is not lost waiting for others to assist with transferring or explaining to patients how to find their allocated cubicle. Conversely though, this may not be an effective use of resources and may actually contribute to inefficiencies in the system, particularly during busy periods (Yang, Lam, Low, \& Ong, 2016). The same could be said for WRN commencing care in cubicles rather than returning immediately to the waiting room. Greater efficiency could potentially be achieved by having the WRN remain in their allocated space, and continuing to assess and commence interventions early.

\section{Methodological strengths and limitations}

Trustworthiness was established. Truth value was established through auditing, confirmation and iteration of the data by the research team to identify codes and then themes to ensure the findings were plausible and reflected the data collected. Collecting data at two different sites increased the applicability of the results. Consistency of quantitative data collection was established 
through face validity testing and pilot study of the observation tool. Potential for observer bias was considered, with the observer adopting an open and honest approach, maintaining confidentiality and privacy both in the setting and in field notes. The observer was conscious and mindful not to impose personal thoughts or assumptions whilst collecting and analysing data (Guba, 1981; Guest, Bunce, \& Johnson, 2006). Over-identification is another potential risk. Becoming too familiar and over-identifying with participants may limit or distort the collected data. In this study data were collected on different days, limiting the time of the sessions and leaving the ED for breaks to minimise this risk (Groenkjaer, 2002).

Two further potential limitations, associated with all observational work are social desirability and observer effect. Social desirability occurs when participants respond in conversations, or their behaviour is influenced during the observation period to ensure they or their performance are viewed favourably by the observer (Schneider et al., 2014). The observer effect transpires when the presence of the observer influences behaviours or activities of participants. This observer effect can be decreased with the development of close relationships with participants and ensuring data are analysed "in light of the context in which they were generated" (Monahan \& Fisher, 2010, p. 363). In this study, these limitations were minimised by the development of meaningful relationships with participants and ensuring that initial periods of observations were passive, focusing on getting to know participants and allowing them to become relaxed in the presence of the observer; the remainder of the observation period then allowed rich data to be collected (Groenkjaer, 2002).

\section{CONCLUSION}

The workload of WRN was observed to be variable and unpredictable, with therapeutic communication and ongoing assessment central to the role. A number of participants perceived experienced, triage prepared emergency nurses as being more efficient in the role, being able to identify and respond to patient and family care needs outside standing orders. It was observed that participants provided holistic, patient centred care to patients and families in the waiting room, primarily through establishing therapeutic relationships and effective communication. WRN contributed to patient safety in the waiting room by commencing episode of care in the waiting room, performing ongoing assessment and management of patients decreasing delays to care and 
detecting patient deterioration, involved patients and families in discussions and worked effectively with interprofessional teams to facilitate care. Some aspects for further consideration in regards to the WRN role include delays in accessing medical officers and appropriate space, re-allocation from the waiting room and limited hours of operation.

\section{RELEVANCE TO CLINICAL PRACTICE}

Therapeutic engagement allowed waiting room nurses to reassure and calm patients and families, and deliver holistic, patient centred care. Waiting room nurses contributed to patient safety in the waiting room, by promptly commencing episodes of care in the waiting room and through close monitoring and assessment to detect patient deterioration.

\section{WHAT DOES THIS PAPER CONTRIBUTE TO THE WIDER GLOBAL CLINICAL}

\section{COMMUNITY?}

- Greater insights into the activities of ED WRN highlights the provision of patient-centred holistic care for patients and families in ED waiting rooms.

- Close engagement and assessment allowed WRN to detect and respond to clinical deterioration early, contributing to safety of patients in waiting rooms.

- Therapeutic engagement and responses by WRN reassured and calmed patients and families in ED waiting rooms.

\section{FUNDING}

We acknowledge the Nurses Memorial Centre, who through the award of a Scholarship, helped enable the postgraduate nursing studies of the first author'. 


\section{REFERENCES}

Asplin, B. R., Magid, D. J., Rhodes, K. V., Solberg, L. I., Lurie, N., \& Camargo Jr, C. A. (2003). A conceptual model of emergency department crowding. Annals of Emergency Medicine, 42(2), 173-180. doi: 10.1067/mem.2003.302

Australasian College for Emergency Medicine. (2013). Policy on the Australasian triage scale (4th ed., pp. 1-3): Australasian College for Emergency Medicine,.

Australasian College for Emergency Medicine. (2016). Statement on night shift rostering of emergency physicians. Retrieved 4th June, 2017, Retrieved from https://acem.org.au/getattachment/69e66298-43dc-4c83-8386-dcd2d4c64e98/S456-

Statement-on-Night-Shift-Rostering-of-Emergen.aspx

Australian Commission on Safety and Quality in Health Care. (2012). National Safety and Qaulity Health Service Standards. Retrieved 8th June 2017, Retrieved from https://www.safetyandquality.gov.au/wp-content/uploads/2011/09/NSQHS-Standards-Sept2012.pdf

Australian Institute of Health and Welfare. (2016). Emergency department care 2015-16. Retrieved 4th July 2017, Retrieved from http://www.aihw.gov.au/WorkArea/DownloadAsset.aspx?id=60129557623

Bernstein, S. L., Aronsky, D., Duseja, R., Epstein, S., Handel, D., Hwang, U., ... Asplin, B. R. (2009). The effect of emergency department crowding on clinically oriented outcomes. Academic Emergency Medicine, 16(1), 1-10. doi: 10.1111/j.1553-2712.2008.00295.x

Boddy, C. R. (2016). Sample size for qualitative research. Qualitative Market Research, 19(4), 426432. doi: 10.1108/QMR-06-2016-0053

Bonner, A., \& Tolhurst, G. (2002). Insider-outsider perspectives of participant observation. Nurse researcher, 9(4), 7-19. doi: https://dx.doi.org/10.7748/nr2002.07.9.4.7.c6194

Borbasi, S., Jackson, D., \& Wilkes, L. (2005). Fieldwork in nursing research: Positionality, practicalities and predicaments. Journal of Advanced Nursing, 51(5), 493-501. doi: 10.1111/j.1365-2648.2005.03523.x

Braun, V., \& Clarke, V. (2006). Using thematic analysis in psychology. Qualitative Research in Psychology, 3(2), 77-101. doi: 10.1191/1478088706qp063oa

Burke, J. A., Greenslade, J., Chabrowska, J., Greenslade, K., Jones, S., Montana, J., . . O'Connor, A. (2017). Two Hour Evaluation and Referral Model for Shorter Turnaround Times in the emergency department. EMA - Emergency Medicine Australasia, 29(3), 315-323. doi: 10.1111/1742-6723.12781

Cecil, P., \& Glass, N. (2015). An exploration of emotional protection and regulation in nurse-patient interactions: The role of the professional face and the emotional mirror. Collegian, 22(4), 377385. doi: https://doi.org/10.1016/j.colegn.2014.06.002

Cheng, I., Lee, J., Mittmann, N., Tyberg, J., Ramagnano, S., Kiss, A., . . Zwarenstein, M. (2013). Implementing wait-time reductions under Ontario government benchmarks (Pay-for-Results): A Cluster Randomized Trial of the Effect of a Physician-Nurse Supplementary Triage Assistance team (MDRNSTAT) on emergency department patient wait times. BMC Emergency Medicine, 13(1). doi: 10.1186/1471-227X-13-17

Clark, P. R. (2009). Teamwork: Building healthier workplaces and providing safer patient care. Critical Care Nursing Quarterly, 32(3), 221-231.

Considine, J. (2011). Emergency department Clinical Initiative Nurse clinical pathway: The Northern Hospital.

Considine, J., Lucas, E., Payne, R., Kropman, M., Stergiou, H., \& Chiu, H. (2012). Analysis of three advanced practice roles in emergency nursing. Australasian Emergency Nursing Journal, 15(4), 219-228. doi: 10.1016/j.aenj.2012.10.001

Creswell, J. (2009). Research Design Qualitative, quantitaive, and mixed methods appraoches (3rd ed.). Singapore: Sage Publications.

Creswell, J., \& Plano Clark, V. (2011). Designing and conducting mixed methods research. Singapore: Sage Publications.

Friberg, K., Husebø, S. E., Olsen, Ø. E., \& Sætre Hansen, B. (2016). Interprofessional trust in emergency department - as experienced by nurses in charge and doctors on call. Journal of Clinical Nursing, 25(21-22), 3252-3260. doi: 10.1111/jocn.13359 
Fry, M., \& Jones, K. (2005). The clinical initiative nurse: Extending the role of the emergency nurse, who benefits? Australasian Emergency Nursing Journal, 8(1-2), 9-12. doi: 10.1016/j.aenj.2005.04.002

Fry, M., MacGregor, C., Ruperto, K., Jarrett, K., Wheeler, J., Fong, J., \& Fetchet, W. (2013). Nursing praxis, compassionate caring and interpersonal relations: An observational study. Australasian Emergency Nursing Journal, 16(2), 37-44. doi: 10.1016/j.aenj.2013.02.003

Fry, M., Ruperto, K., Jarrett, K., Wheeler, J., Fong, J., \& Fetchet, W. (2012). Managing the wait: Clinical initiative nurses' perceptions of an extended practice role. Australasian Emergency Nursing Journal, 15(4), 202-210.

Garling, P. (2008). Final Report of the Special Commission of Inquiry Acute Care Services in NSW Public Hospitals (Vol. 3). N.S.W.: State of NSW through the Special Commission of Inquiry: Acute Care Services in New South Wales Public Hospitals

Gold, R. (1958). Roles in sociological field observations. Social Factors, 36(3), 217-223.

Groenkjaer, M. (2002). Critical ethnographic methodology in nursing research: issues and solutions. Contemporary nurse : a journal for the Australian nursing profession, 14(1), 49-55.

Guba, E. G. (1981). Criteria for assessing the trustworthiness of naturalistic inquiries. Educational Communication \& Technology, 29(2), 75-91. doi: 10.1007/BF02766777

Guest, G., Bunce, A., \& Johnson, L. (2006). How many interviews are enough? an experiment with data saturation and variability. Field Methods, 18, 59-82. doi: 10.1177/1525822X05279903

Huang, E. P. C., Liu, S. S. H., Fang, C. C., Chou, H. C., Wang, C. H., Yen, Z. S., \& Chen, S. C. (2013). The impact of adding clinical assistants on patient waiting time in a crowded emergency department. Emergency Medicine Journal, 30(12), 1017-1019. doi: 10.1136/emermed-2012-201611

Hubner, P., Schober, A., Sterz, F., Stratil, P., Wallmueller, C., Testori, C., . . Weiser, C. (2015). Surveillance of patients in the waiting area of the department of emergency medicine. Medicine (United States), 94(51). doi: 10.1097/MD.0000000000002322

Innes, K., Jackson, D., Plummer, V., \& Elliott, D. (2015). Care of patients in emergency department waiting rooms - an integrative review. Journal of Advanced Nursing, 71(12), 2702-2714. doi: 10.1111/jan.12719

Innes, K., Jackson, D., Plummer, V., \& Elliott, D. (2017). Emergency department waiting room nurse role: A key informant perspective. Australasian Emergency Nursing Journal, 20(1), 6-11. doi: 10.1016/j.aenj.2016.12.002

Jackson, D., McDonald, G., Luck, L., Waine, M., \& Wilkes, L. (2016). Some strategies to address the challenges of collecting observational data in a busy clinical environment. Collegian, 23(1), 47-52. doi: 10.1016/j.colegn.2014.10.001

Kamali, M. F., Jain, M., Jain, A. R., \& Schneider, S. M. (2013). Emergency department waiting room: Many requests, many insured and many primary care physician referrals. International Journal of Emergency Medicine, 6(1). doi: 10.1186/1865-1380-6-35

Luck, L., Jackson, D., \& Usher, K. (2009). Conveying caring: Nurse attributes to avert violence in the ED. International Journal of Nursing Practice, 15(3), 205-212. doi: 10.1111/j.1440172X.2009.01749.x

Mason, S., Knowles, E., \& Boyle, A. (2017). Exit block in emergency departments: A rapid evidence review. Emergency Medicine Journal, 34(1), 46-51. doi: 10.1136/emermed-2015-205201

Monahan, T., \& Fisher, J. A. (2010). Benefits of 'observer effects': Lessons from the field. Qualitative Research, 10(3), 357-376. doi: 10.1177/1468794110362874

Morse, J. M. (2003). Perspectives of the Observer and the Observed. Qualitative Health Research, 13(2), 155-157. doi: 10.1177/1049732302239595

Mulhall, A. (2003). In the field: Notes on observation in qualitative research. Journal of Advanced Nursing, 41(3), 306-313. doi: 10.1046/j.1365-2648.2003.02514.x

Murphy, E., \& Dingwall, R. (2007). Informed consent, anticipatory regulation and ethnographic practice. Social Science and Medicine, 65(11), 2223-2234. doi: 10.1016/j.socscimed.2007.08.008

National Health and Medical Research Council. (2007). National Statement on Ethical Conduct in Human Research. ACT: Australian Government.

Odell, J. (2015). Clinical decision making in minor illness. Practice Nursing, 13, 504-507.

Rehnström, K., \& Dahlborg-Lyckhage, E. (2016). Proactive Interventions: An Observational Study at a Swedish Emergency Department. SAGE Open, 6(3). doi: 10.1177/2158244016658083 
Schneider, Z., Whitehead, D., Lobiondo-Wood, G., \& Haber, J. (2014). Nursing and midwifery research methods and apparaisal for evidence-based practice (5th ed.). NSW: Elsevier.

Smyth, O., \& McCabe, C. (2016). Think and think again! Clinical decision making by advanced nurse practitioners in the Emergency Department. International Emergency Nursing. doi: 10.1016/j.ienj.2016.08.001

Tashakkori, A., \& Teddlie, C. (1998). Mixed methodology: combining qualitative and quantitaive approaches. London: Sage Publications.

Turnock, C., \& Gibson, V. (2001). Validity in action research: A discussion on theoretical and practice issues encountered whilst using observation to collect data. Journal of Advanced Nursing, 36(3), 471-477. doi: 10.1046/j.1365-2648.2001.01995.x

Welch, S. J. (2010). Twenty years of patient satisfaction research applied to the emergency department: A qualitative review. American Journal of Medical Quality, 25(1), 64-72. doi: $10.1177 / 1062860609352536$

World Health Organization. (2013). Health literacy the solid facts. Europe: World Health Organisation.

Yang, K. K., Lam, S. S. W., Low, J. M. W., \& Ong, M. E. H. (2016). Managing emergency department crowding through improved triaging and resource allocation. Operations Research for Health Care, 10, 13-22. doi: 10.1016/j.orhc.2016.05.001 